Community Nursing Quality Indicators for End of Life Care in England 2014-15:

A Campaign for Identification, Preparation and Coordination

Jane Cook

Clinical Community Nurse Advisor (QuICN Project)

University of the West of England, Glenside Campus, Blackberry Hill,

Stapleton, Bristol, BS16 1DD.

Jcook@nhs.net

0117-9710928

Susan Horrocks, Senior Lecturer, University of the West of England, Bristol.

Total Word Count: 3, 526 including table and references

Article: 2, 555

\title{
Funding Acknowledgement:
}

This project was funded by the National Institute for Health Research:

Measuring Quality in Community Nursing: A mixed methods study HS\&DR 12/209/02

\section{Department of Health Disclaimer:}

The views and opinions expressed therein are those of the authors and do not necessarily reflect those of the NIHR, NHS or the Department of Health. 


\begin{abstract}
:
High quality community nursing is essential to ensure that end of life care can be provided in community settings, in line with patient preferences. This article seeks to examine what quality priorities commissioners sought to incentivise in end of life care, by reviewing a survey of Commissioning for Quality and Innovation indicators for community nursing conducted in England, 2014-15. Emerging findings from the survey suggest that end of life care did not have a high priority with the CQUIN indicators for community nursing. Vigorous quality standards, including training and development, need to be in place to make sure that the potential of community nursing is utilised to sensitively engage with people nearing the end of their lives and support them to plan their future care, if they wish to.
\end{abstract}

\title{
Five Key Points:
}

- A well-trained and resourced community nursing workforce has the potential to support more people to die at home, if it is their wish.

- CQUIN indicators for community nursing in England in 2014-15, which incentivise areas for service development and improvement, did not place a high priority on end of life care.

- Themes in end of life CQUIN indicators for community nursing included earlier identification of people nearing the end of their lives, advanced care planning and coordination and communication between services involved in end of life care.

- There was an over-emphasis on activity measurement within CQUIN indicators, rather than on the quality and experience of care provided.

- Quality initiatives need to incorporate provision for staff training and development to support community nurses to confidently identify more people approaching the end of their lives, and support care-planning in line with their wishes and preferences.

Keywords: Quality, CQUIN, End of Life Care, Community Nursing, Advanced Care Planning 


\section{Background}

Identification of more people at the end of their lives and particularly those with non-cancer diagnoses, like chronic disease, dementia and frailty, is an important NHS initiative (NICE, 2011). A recent report, 'Dying without Dignity' highlighted a series of case studies where clinicians failed to recognise when people were dying and to proactively plan to manage their care needs, leading to unnecessary distress and avoidable crises (Parliamentary and Health Service Ombudsman, 2015). The National Primary Care Snapshot Audit found that only $27 \%$ of the people who died were included on the GP's palliative care register, where their care could be planned and co-ordinated in advance (Thomas et al, 2009). This has prompted campaigns like 'Find your $1 \%$ ' where GPs are encouraged to identify more of their practice population likely to die within the next year to enable appropriate end of life advance care planning (Dying Matters Coalition, 2011).

While identification and advance care planning undoubtedly constitute best practice (NICE, 2011), there has been much controversy about the methods adopted to achieve these ends. Headlines about singling out frail, elderly people to be put on 'Death Lists' in primary care, and incidences of people being cold-called to ask them if they wanted resuscitation has fuelled public concerns that plans about their future care were being made without proper consultation (Borland, 2015, Doughty, 2012). A review of the Liverpool Care Pathway highlighted that people and their carers were not always routinely being involved in decision-making about the transition of treatment to palliative care (Neuberger et al, 2013).

Provision of end of life care (EoLC) is a core function of the district nursing services (Department of Health, 2013). Studies examining how community nurses provide effective palliative care to people at home have highlighted the importance of developing a relationship with patients and carers to support their care (Washe \& Luker, 2010, Nagington et al, 2013). It is likely that earlier identification of people nearing the end of their lives could help establish 
rapport (Dunne et al, 2005). However, there is little research guidance on what could improve community nurse practice in terms of patient experience and outcomes in this area.

Community nurses could be well-placed to broach issues like planning for future care in a sensitive way as they support the care needs of many frail, elderly, housebound people on their caseloads, who may be nearing the end of their lives. Although most people would prefer to die in their own home, less than a fifth are able to do so, particularly those with non-cancer diagnoses (While, 2012). Earlier identification by community nurses and sensitive conversations about preferences and wishes, enabling care planning, could be an opportunity to redress this disparity. There is evidence of the success of community service developments to facilitate people being able to die in their own home (Wye et al, 2014). However, nurses often lack confidence and training in raising these issues with patients and their families and can struggle with pyscho-social aspects of palliative care (House of Commons Health Committee, 2015, Keogh, 2014, Walshe \& Luker, 2010).

It is clear that indicators of high quality service around identification of those nearing the end of their lives and their future care planning should reflect the context and the sensitivity of how these issues are introduced and discussed with people, and not just be about targeting greater numbers. This article examines emerging findings from a survey of Commissioning for Quality and Innovation (CQUIN) indicators for community nursing in England in 2014-15 (Horrocks et al, 2015 unpublished report), particularly focusing on indicators for EoLC. It also looks at how such quality initiatives, national and local, filter down to frontline community nursing. 


\section{Commissioning for Quality and Innovation - National Survey}

The Commissioning for Quality and Innovation (CQUIN) framework is used by commissioners to influence healthcare providers to implement quality standards and service improvement. The framework is a powerful lever because CQUIN link a proportion of the provider's income to the delivery of quality targets within a designated time-frame, usually over a year (Olphert, 2015). Robust quality measures need to be in place to ensure that identification and planning of care for people nearing the end of their lives is undertaken compassionately and in accordance to their needs and wishes, and that on-going care is supported after identification.

A proportion of CQUIN indicators are set nationally and were mandatory for NHS service providers in 2014-15. Others are decided locally. Commissioners and providers agree shared aims and aspirations for service development, which are then translated into goals for local CQUINS. Each CQUIN indicator comprises a stated goal or aspiration to improve a service and an intended means of measurement to demonstrate that the goal has been achieved. One hundred and fifty nine clinical commissioning groups (75\% of all the CCGs in England) sent details of CQUIN indicators to the research team in a survey conducted in England in 2014-15. Three researchers with professional experience of either community nursing or commissioning collaborated to produce coding schemes and to code the data. All the CQUINS received were coded for the following factors:

- Applicability to community nursing

- Type of indicator (national or local)

- Area of care addressed (e.g. dementia)

Resulting data were descriptively analysed using SPSS Statistics 20.0 with content analysis of the most frequent CQUIN goal themes.

In total, 889 indicators (74\% of all the indicators submitted) were judged to definitely apply to community nursing. These indicators were then divided into national or local CQUIN (see Figure 1). National indicators allow comparisons 
to be made across different areas of England, so that an aspect of care can be standardised nationally and progress reviewed. The Friends and Family Test and the National Safety Thermometer were national CQUINs for community providers in 2014-15. There were no national CQUIN indicators applying to EoLC. This could be construed as a missed opportunity as it is known that there are wide geographical variations in the provision of EoLC (While, 2012).

The local CQUIN indicator goals were categorised into 13 care themes (see Figure 1). Organisation of care and organisational issues accounted for $33 \%$ of the local CQUIN indicators, making this a dominant theme. Only thirty-six local CQUIN indicators (7\% of all local CQUIN indicators) related specifically to EoLC. EoLC did not seem to be a high priority within the CQUIN framework.

Figure 1: Breakdown of Analysis of CQUIN Indicators 2014-15

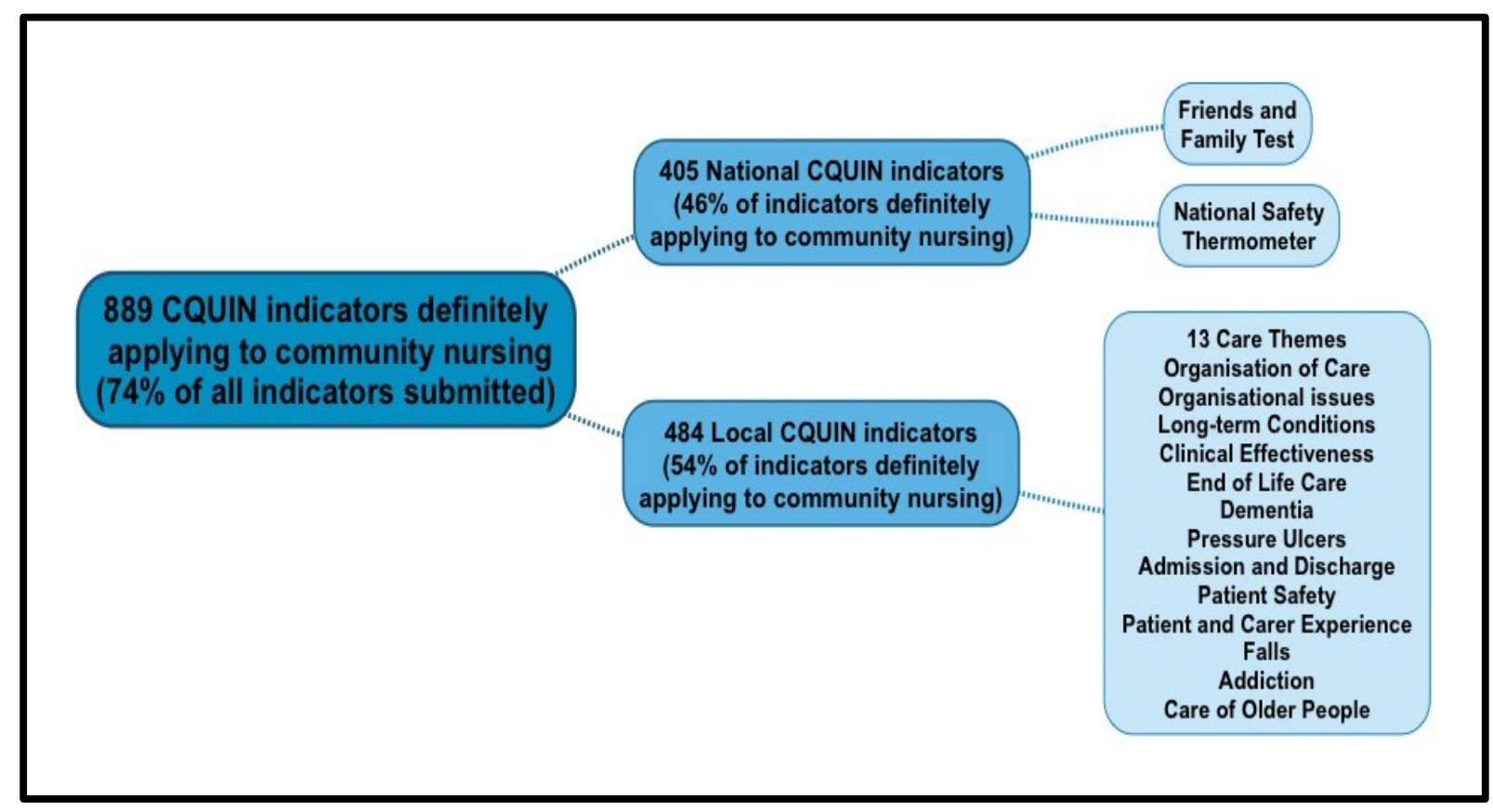

Two researchers undertook further analysis of the thirty-six local end of life CQUIN indicators, focusing on the intended goal and the milestones required to achieve the indicator. These were categorised into sub-themes to identify 
what aspects of EoLC were important to incentivise at a local level. Three principal sub-themes emerged (see Figure 2 below).

- Identification of more people nearing the end of their lives

- Advanced care planning

- Coordination and collaboration between services involved in EoLC.

Figure 2: Sub-themes in Local End of Life CQUIN Indicators

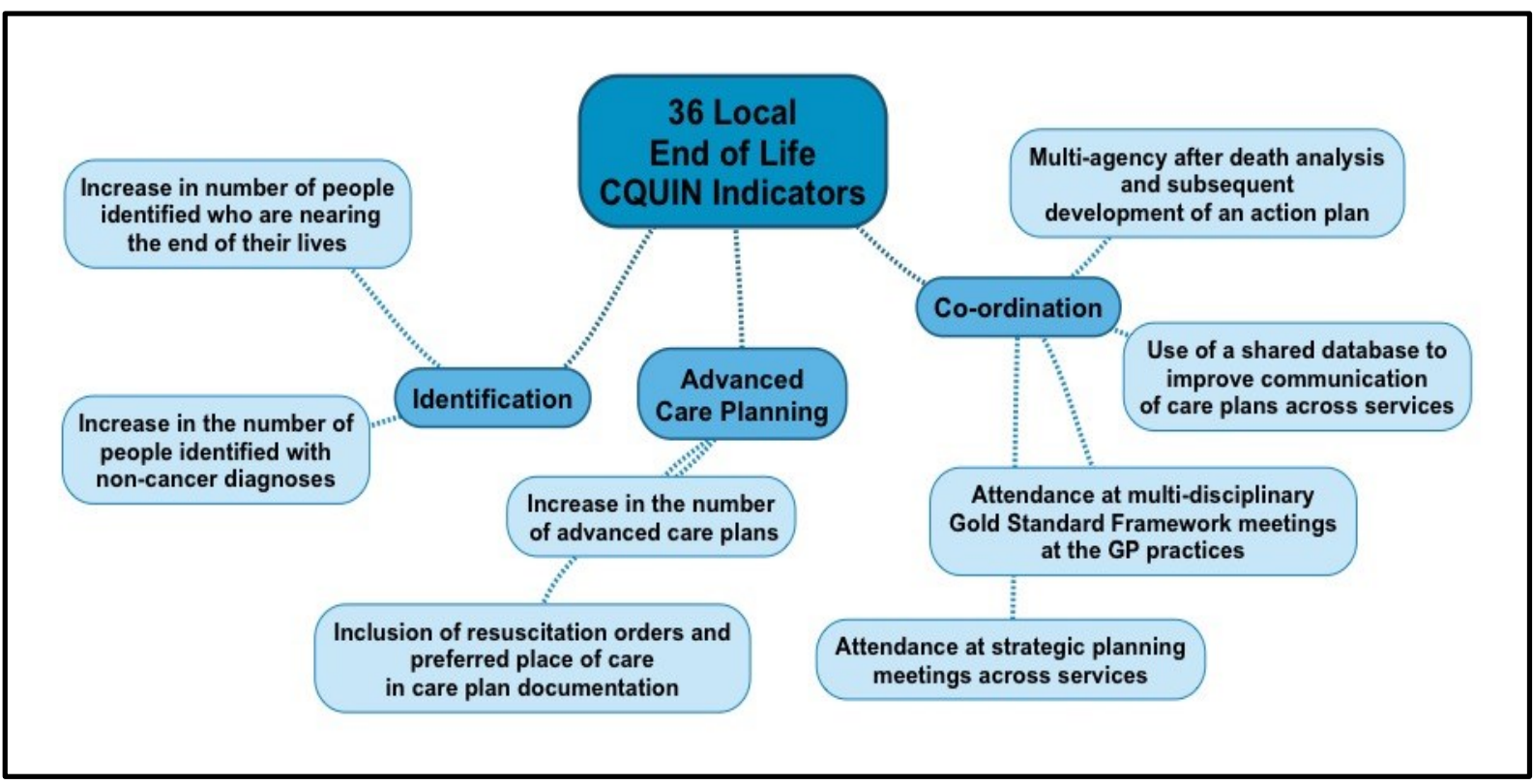

Over a third of the CQUIN indicators used activity measurement criteria focused on increasing the numbers of people identified as being 'end of life' and numbers of care plans. A minority of CQUINs tried to capture information about whether these strategies resulted in improved patient and carer experience. However, it is possible that this was being collected through different mechanisms alongside the CQUIN indicators. 


\section{Discussion}

It would appear from emerging findings of the survey of CQUIN indicators for community nursing in 2014-15 that EoLC was not considered a high priority for community nursing. No national CQUIN indicators and only $7 \%$ of local CQUINs focused on palliative care. This is surprising when there is evidence that $10-20 \%$ of the NHS budget is spent on people in their last year of life, largely due to hospital admissions (Palliative Care Funding Review, 2011) and that people with access to community-based nursing are more likely to die at home than the rest of the population (National Council for Palliative Care, 2013). The House of Commons Health Committee (2015) similarly highlighted that EoLC was not being treated as core business for the NHS, because of the lack of EoLC direction provided in the Five Year Forward View. The National Council for Palliative Care (2013) indicated that fewer than half of local Health and Wellbeing boards had explicitly considered dying people in their strategy, suggesting that there is a need for strong leadership to ensure EoLC is embedded into mainstream NHS planning at all levels.

Within the end of life CQUIN indicators, there appeared to be an overemphasis on activity targets, involving increasing the numbers of patients identified or care plans completed, rather than on assuring the quality and experience of the care provided. The National Council for Palliative Care (2013) highlighted the lack of a universally recognised outcome measure for EoLC, enabling an individual's quality and experience of care to be assessed and allowing services and localities to be benchmarked. The National Survey of Bereaved People (VOICES) is commissioned by NHS England to independently collect information from bereaved people in England on their perception of care provided to a friend or relative in the last 3 months of life (Office of National Statistics, 2015). This allows for comparison of regional variation of carer experience and informs national end of life strategy, but cannot indicate the local impact of a single quality improvement (like a CQUIN indicator) on clinical effectiveness of community nursing or the impact on patient experience of earlier identification. 
For the community nurses working in practice, earlier identification of patients who may be at the end of their lives and discussion about their preferences for care is fraught with difficulty. Patients may not consider themselves as end of life and there is potential to cause psychological distress, which is particularly worrying for nurses if scarce resources make it hard to support ongoing pyscho-social needs. It remains unclear whether information of impending death makes a significant difference to patients' quality of life, even it is true that they are able to plan their care (Nyatanga, 2015). Advanced care planning is legally complex, with documentation that staff may not be familiar with (Griffiths, 2014). The House of Commons Health Committee (2015) recommended that all staff providing EoLC should receive training in communication skills and advance care planning. Only a few of the CQUIN indicators referred to training programmes to support staff with identification and care planning. One indicator, in particular, links a regional training programme about shared decision-making and advanced care planning with activity to offer patients with long-term conditions or frailty on community nursing caseloads an opportunity to discuss their preferences for care. The indicator encourages nurses to think more broadly about who might be nearing the end of life within their own sphere of influence, while giving them to skills and knowledge to broach a sensitive subject in more abstract terms in the context of choice.

With regard to advanced care planning, there are valid and ethical concerns that resources are not available for patients to have real choice about their care provision. A RCN survey found that $70 \%$ of community nurses had experienced people being admitted to hospital in their final hours, against their wishes, because there were in sufficient resources to care for them at home (Keogh, 2014). There are difficulties in accessing care packages and night sitting at the right time. There has also been a $44 \%$ reduction in the number of qualified district nurses in England in the last decade, in contrast to an aging demographic increasing the number of people with complex life-limiting conditions needing nursing care in the community (Ball et al, 2014). Quality measures need to examine the effect of these shortfalls on the ability of community nursing to support patients not only to plan their care but also to 
achieve their preferences, particularly as with greater identification, more patients will be encouraged to make plans for their future care.

Using the pattern of local CQUINs for EoLC to measure priorities for quality of the community nurse role can only offer a partial perspective. Commissioners may use other contractual levers to ensure high quality provision, such as local key performance indicators. Nationally, the Care Quality Commission (CQC) now routinely inspects EoLC in community providers to monitor standards of practice, incorporating caring and responsiveness as aspects of quality (CQC, 2015). There is a plethora of national guidance, frameworks and recommendations to support local CCGs planning for EoLC, including NICE Quality Standards (NICE, 2011), the Five Priorities for Care replacing the Liverpool Care Pathway (Leadership Alliance for the Care of Dying People, 2014), and most recently the refreshed End of Life Strategy encompassing six ambitions for EoLC (National Palliative and End of Life Care Partnership, 2015). However, while there is strong national encouragement to take up these initiatives, their adoption is not mandatory for local CCGs and their permeation into frontline community nursing care is unclear, particularly if powerful levers like the CQUIN framework are not being used as incentives.

There is little understanding or acknowledgement of the skills and knowledge base of community nurses in EoLC. They work in less visible, home-settings with patients who do not have a strong, unified voice and whose outcomes are hard to identify because their health is generally so poor. There is a pressing need for community nurses to raise their profile in regard to palliative care, so that they can positively influence decisions about the quality and the development of the service. Walshe and Luker (2010) highlighted three areas where further investigation is needed: observation of district nurse practice, outcomes-focused work and examination of the views of patients and carers about the processes and outcomes of care. All of these need commitment and support from frontline community nurses and can help to determine standards to demonstrate quality in community nursing EoLC. Nurses also need to actively seek out opportunities to become involved in commissioning to 
ensure that EoLC is given a high priority in how their service is developed. The CQUIN survey is part of a wider study examining how the quality of community nursing is defined and measured in five case study sites and who is involved at different stages, to gain a better understanding of the process. The progress of the study can be accessed at www.quicn.uk/.

\section{Conclusion}

The role of community nurses in EoLC is crucial to supporting more people to die at home, if they wish to do so. Quality initiatives need to assist community nurses to have the confidence and skills to engage in meaningful and sensitive conversations about peoples' end of life wishes and preferences for future care. Although, EoLC was not a high priority for community nursing within the CQUIN framework, the landscape of quality measurement in EoLC is a complex one with many independent strands, both nationally (including the VOICES survey, CQC, the quality initiatives) and locally. There are concerns that these elements are not always being translated into mainstream NHS planning, where they could have the greatest effect for statutory services. Community nurses need to raise awareness of what they do in EoLC to ensure that they are able to influence decision-makers to progress the expansion and development of their role in this area. Quality measurement for community nursing in EoLC should be rigorous enough to capture how interventions are delivered, how they benefit patients and carers experiences of care and their effectiveness in terms of patient outcomes.

\section{Reference List:}

Ball J, Philippou J, Pike G, Sethi G (2014) Survey of District and Community Nurses in 2013: Report to the Royal College of Nursing. NNRU. London. 
Borland S (2015) Over 75? Sign up here if you are ready for death. The Daily Mail [on-line] 26 April. Available at < http://www.dailymail.co.uk/news/article3056621/Over-75-Sign-ready-death-GPs-ask-older-patients-II-agree-notresuscitate-order.html> [Accessed on 10 September 2015].

Care Quality Commission (2015) Themed Review of End of Life care. CQC [on-line] Available at < http://www.cqc.org.uk/content/themed-review-end-lifecare> [Accessed on 23 October 2015].

Department of Health (2013) Care in Local Communities: A New Vision and Model for District Nursing. DoH. London.

Doughty S (2012) Three thousand doctors putting patients on death lists that single them out to be allowed to die; The Daily Mail [on-line] 18 October Available at < http://www.dailymail.co.uk/health/article-2219351/ThreeTHOUSAND-doctors-putting-patients-death-lists-single-allowed-die.html> [Accessed on 10 September 2015].

Dunne K, Sullivan K, Kernohan G (2005) Palliative Care for Patients with Cancer: District Nurses Expereinces. Journal of Advanced Nursing 50 (4) pp372-380. Doi: 10.1111/j.1365-2648.2005.03402.

Dying Matters Coalition (2011) Find Your 1\% Campaign [on-line] London. National Council for Palliative Care. Available at <:https://www.Dyingmatters.org/gp page/about-campaign> [Accessed 10 November 2015].

Griffiths R (2014) District Nurse Consultations with Vulnerable patients over wishes for end of life care. Br J Community Nurs. 19 (9):459-460. doi.org/10.12968/bjcn.2014.19.9.458

Horrocks S, Pollard K, Duncan L, et al (2015) Measuring Quality in Community Nursing: A Mixed Methods Study; 
A Report on Emerging Findings from a National Survey of CQUINs Applying to Community Nursing (unpublished)

http://www.quicn.uk/pdfs/Emerging\%20Findings\%20of\%20CN\%20CQUIN\%2 0Survey\%20Jan\%2015.pdf [Accessed July 2015].

House of Commons Health Committee (2014-15) End of Life Care Fifth Report of Session 2014-15. The Stationary Office. London

Keogh K (2014) RCN Survey reveals concerns over end of life care. Nursing Standard. 29 (9). doi.org/10.7748/ns.29.9.0.299991

Leadership Alliance for the Care of Dying People (2014) One Chance to Get it Right. DoH. London.

Nagington M, Luker K, Walshe C (2013) Busyness and the Preclusion of Quality Palliative District Nursing Care. Nursing Ethics. 20 (8) pp893-903. Doi: 10.1177/0969733013485109.

National Council for Palliative Care (2013) The End of Life Care Strategy: New Ambitions. National Council for Palliative Care. London.

National Institute for Health and Care Excellence (2011) NICE Quality Standard [QS-13]: Quality Statement 1: Identification [on-line] London. NICE. Available at < https://www.nice.org.uk/guidance/qs13/chapter/QualityStatement-1-identification > [Accessed on 12 October 2015].

National Palliative and End of Life Care Partnership (2015) Ambitions for Palliative and End of Life Care: A National Framework for Local action 20152020. NHS Gateway. London .

Neuberger J, Guthrie C, Aaronvitch D et al (2013) More Care, Less Pathway: A Review of the Liverpool Care Pathway. DoH. London. 
Nyatanga B (2015) Knowledge of Impending Death and its effect on the Quality of Dying. Br J Community Nurs 20 (3):153.

doi.org/10.12968/bjcn.2015.20.3.152.

Office for National Statistics (2015) National Survey of Bereaved People 2014 (VOICES). DoH. London

Olphert A (2015) Commissioning: CQUINs. Br J Nurs. 24 (5): 298;

doi/abs/10.12968/bjon.2015.24.5.298.

Palliative Care Funding Review (July 2011) Funding the Right Care and Support for Everyone. DoH. London.

Parliamentary and Health Service Ombudsman (2015) Dying without Dignity: Investigations by the Parliamentary and Health Service Ombudsman into Complaints about End of Life Care. DoH. London.

Thomas, K, Clifford C, de Silva D et al (2009-10) The National Primary Care Snapshot Audit in End of Life Care, England 2009-10. DoH. London.

WalshC, Luker K (2010) District Nurses Role in Palliative Care Provision: A Realist Review. International Journal of Nursing Studies. 47 pp1167-1183. doi:10.1016/j.ijnurstu.2010.04.006.

While A (2012) Getting End of Life Care Right. Br J Community Nurs.17 (11): 556. doi/10.12968/bjcn.2012.17.11.556

Wye L, Lasseter G, Percival J, Duncan L, Simmonds B, Purdy S (2014) What Works in 'Real Life' to Facilitate Home Deaths and Fewer Hospital Admissions: Results from a Realist Evaluation of New Palliative Care Services in Two English Counties. BMC Palliative Care.13 (37) doi:10.1186/1472-684X-13-37 